\title{
Positive Mental Health, Need for Holistic Approach
}

\author{
Sharma PP
}

Mental health is not just the absence of mental disorder, but is a state in which an individual has a sense of well being in which he or she realizes his or her own potential, can cope with the normal stresses of life and thus can work productively and fruitfully and is able to contribute to their own community. Mental illness is not uncommon with approximately 450 million people affected globally according to WHO estimate. ${ }^{1}$ However, there is no credible data available on the burden of mental health in Nepal as yet. It is prevalent in all population groups, however those with psychosocial stressor such as poverty and unemployment are particularly at risk. Mental illness is also commonly associated with long term disability and is manifest with decrease in both physical and social functioning. Regarding the morbidity, prevalence studies alone does not portray the holistic picture of the impact of mental illnesses on the society. The effect of the illness on the society is reflected by the burden of care to the family, society, and the community which signifies its importance of treating, rehabilitating, and preventing mental illness. It is a well known fact that positive mental health is linked to a range of developmental outcome and is fundamental in coping with adversity. On the other hand, poor mental health is responsible for reducing persons' capacity to realize their potential, work productivity and contribute to the community.

Unfortunately mental health problem always receives less priority in terms of resource allocation in Governmental as well as the Nongovernmental organizations as the entire priority is given to communicable diseases in low income countries and are more focused on the fatal illnesses such as cancer and cardiovascular illnesses in high income countries. As less funding is available for mental health promotion aimed at having a positive effect on mental well being, encouragement of individual resources, skills and improvement in socio-economic environment is one among the strategies used.

Common mental disorders like depression are one of the leading causes of disability and the fourth leading contributor to the Global Burden of Disease (GBD). By the year 2020, depression is projected to reach second place in the ranking of Disability Adjusted Life Years (DALY) calculated for all ages, both sexes combined. ${ }^{1}$ Suicide is another common and severe mental health problem in the community and about one million people die from suicide every year globally. ${ }^{1}$ There are abundance amount of literatures showing that mental disorders interact with somatic health conditions both in general population and medical care setting. ${ }^{2,3}$ Cardiovascular diseases have strong prospective relation with depression and anxiety. ${ }^{4}$ Depression increases the risk of type II diabetes ${ }^{5}$ and some indirect evidence suggests that people with mental disorder are at higher risk for contracting HIV. ${ }^{6}$ There are multitude of other mental health problems that can be treated or prevented there by improving the quality of life of large number of individuals, families and the society as a whole. Combination of social, psychological and pharmacological approaches in the treatment of mental illness is cost effective.

Lack of adequate mental health facilities in the community, lack of awareness among individuals, society and the country as a whole is taking a heavy toll in the lives of general population in terms of social, mental, and financial burden. Paucity of resources in mental health along with other socio-economic factors playing negative role in the delivery of mental health care could be alleviated by incorporating mental health into the primary health care system and by encouraging Private Public Parternership (PPP). If government can assume a proactive role to bring these private institutions into the mainstream by encouraging Private Public Partnership (PPP), a lot more can be achieved. Locally available resources like community leaders, school teachers, social workers and religious leaders can be mobilized to achieve the target in the 
promotion and prevention of mental health. Often ignored but important fact about the mental illness is that there is a tendency to overlook the complexity of any specific mental heath problem which arises from and leads to the individual, the family and the society at large. This burden of care has to be addressed effectively by managing mental illness at the community level as well. Family members should also be involved in the management of mental health problems.

Stigma attached to mentally illness is one more serious hurdle in delivering mental health service to the community. Stigma brings about shame, social exclusion, isolation and many other problems to mentally ill person and their families that discourage them to utilize available mental health services resulting in poor compliance and poor outcome. People with mental illness in Nepal experience significant stigma during their course of illness and treatment and is associated with relapse of symptoms and non compliance of treatment. ${ }^{7}$

It is very important to obtain appropriate national data on the burden of mental health without much of a delay and integrate mental health services with all aspects of health and social policy, health system planning and delivery of primary and secondary health care in the country. We must accept the fact that there is no health without mental health.

\section{REFERENCES}

1. World Health Organization [Internet]. What is mental health? [updated 2007 Sep 3; cited 2010 Dec 9]. Available from: http://www.who.int/features/qa/62/en/

2. Kroenke K, Prince K. Symptoms in the community, prevalence, classification and psychiatric commodity. Arch Interp Med 1993; 153: 2474-80.

3. Kronke K, Spitzer RL, William JB et al. Physical Symptoms in primary care. Predictors of psychiatric disorders and functional impairments. Arch Fam Med 1994;3:774-79.

4. Hemingway H, Marmot M. Evidence based Cardiology Psychosocial factors in the etiology and prognosis of coronary heart disease. System review of prospective Cohort studied. BMJ 1999;318:1460-67.

5. Eaton WW, Armenian H, Gallo J, Pratt L, Ford DE. Depression and risk for onset of type II diabetes. A prospective population-based study. Diabetes Care 1996;19:1097-102.

6. Ciesla JA, Roberts JE. Meta-analysis of the relationship between HIV infection and risk for depressive disorders. Am J Psychiatry 2001; 158:725-30.

7. Adhikari S, Pradhan SN. Experiencing stigma: Nepalese prospective. Kathmandu Univ Med J 2008;6(4): 45865. 